\title{
Study of Model Data Exchange Method Between Different Structure CAD Systems
}

\author{
Qian Ding \\ College of Management \\ Harbin University of Science and Technology \\ Harbin, P.R.China \\ 13351985596@163.com
}

\author{
Changzheng Liu \\ College of Computer Science and Technology \\ Harbin University of Science and Technology \\ Harbin, P.R.China \\ liuchangzhengy@163.com
}

\begin{abstract}
A novel data exchange method based on neutral semantic feature conversion was proposed in this paper. $\mathrm{A}$ neutral semantic feature converter was used as transfer station, and modeling information of different systems would be transformed into a kind of unified data message format, and feature dependency graph was used to represent feature modeling information. Mapping relations between heterogeneous CAD systems' commands were implemented using heterogeneous semantic conversion based on this method. System implemented in this paper indicated that synchronous collaborative system of heterogeneous CAD proposed can make users work in a environment of real-time collaborative, and can improve product quality and reduce product design cycle.
\end{abstract}

Keywords- CAD, data exchange, semantic feature modeling, neutral semantic

\section{INTRODUCTION}

With the application and development of network technology, traditional stand-alone CAD system with single-user mode have been expanded to network CAD system. Development of network CAD system make a group can complete a modeling work cooperatively in the environment of computer technology supporting, and it can shorten development cycle and reduce product development costs. But research on network CAD system, especially on synchronous collaborative[1][2] between heterogeneous CAD systems is still at the primary stage, and most platform design between heterogeneous CAD systems only support data transmission of single feature and it is difficult to transfer semantic information and design intent between heterogeneous CAD systems. So it results in not only product development cycle extension, but also CAD modeling design low quality based on network.

In view of the above questions, a data exchange method based on neutral semantic feature conversion is proposed in this paper. A neutral semantic feature converter is used as transfer station in this method, and modeling information of different systems will be transformed into a kind of unified data message format in the transfer station, and feature dependency graph[3] in semantic feature modeling technology[4][5] is used to represent feature modeling information. Feature dependency graph is a kind of declaring feature modeling technology[6][7], and it can overcome some shortcomings in traditional modeling technology based on history model[8], can keep high-level semantic information, and can describe accurately the designer's design intent.

Mapping relations between heterogeneous CAD systems' commands are implemented using heterogeneous semantic conversion based on data transform method of neutral semantic feature transition. Based on this mapping relations, synchronous collaborative transmission of single part is realized, so this method supports effectively view consistency between different systems and achieves synchronous collaborative design effects named "what you see is what I see" between heterogeneous CAD systems.

\section{DATA EXCHANGE METHOD BASED ON NEUTRAL SEMANTIC FEATURE}

At present, data exchange method in collaborative design has two types: data exchange based on geometric and feature parameter exchange based on parameter feature[6].

Data exchange method based on geometric is a kind of data exchange at the level of model boundary representation or surface patch representation. Because feature model is usually complex and data volume is also very large, the method that transforms directly object model must reduce severely transmission efficiency and response speed of modeling system. So this method is only used in asynchronous collaborative design system.

Most design system are parameterization design system based on feature, so model data exchange are achieved by exchanging structure parameters of features in the process of collaborative design, and this method has smaller transmission size than the one based on geometric exchange. Because of various kinds of commercial factors, there is not a unified standard in the field of modeling command and feature internal structure in today's design system, so it caused some difficult in the application of feature parameter exchange based on heterogeneous system.

A kind of data exchange method based on neutral semantic feature conversion is proposed on the basis of parameter feature in this paper. Features dependence graph is used to present unify modeling information, and this 
method support not only single feature's transmission, but also single part's transmission, synchronous collaborative design between heterogeneous CAD systems is achieved in this way.

\section{A. neutral semantic feature converter}

Feature models are constructed by semantic feature modeling based on cellular represent, and data exchange between different systems are implemented by neutral semantic feature converter.

When user executes a modeling command LSMC in a local CAD system, the command is transformed into neutral semantic feature converter. System search and finds a feature class matched with the command in feature class library, and a neutral semantic feature converting object is generated. Feature dependence graph is used to save feature information in the neutral semantic feature convertion command NSFT. The command was sent ot master site, and after successful validation, a accepted successfully message is sent to slave site, and the NSFT command is sent to other slave sites. After other slave sites receive the NSFT command, the command is transmitted to neutral semantic feature converter, the command is transformed to LSMC command which is recognized by itself and then is executed. This process is shown in Fig.1.

site 1

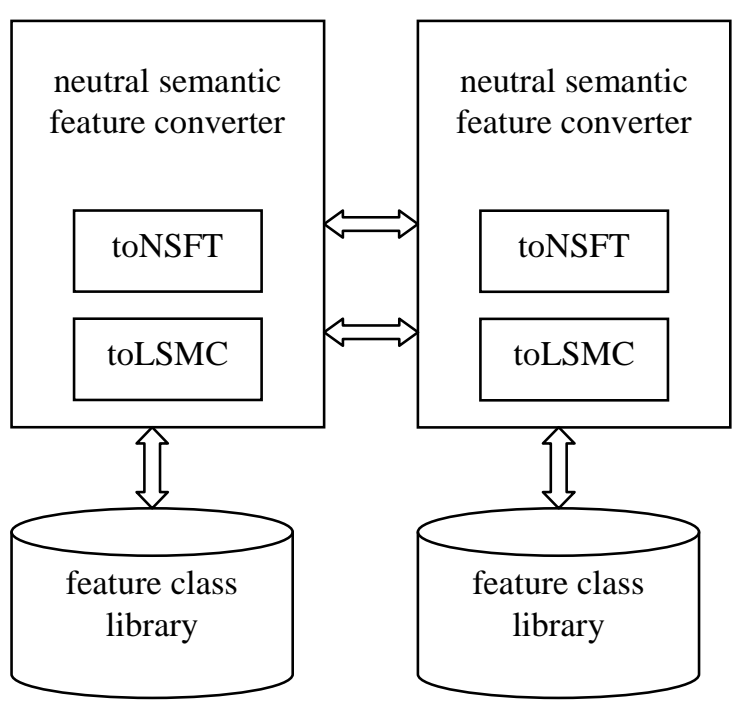

Figure 1. data exchange flow between different sites

Neutral semantic feature converter is mainly composed of toNSFT( ) and toLSMC(), function toNSFT() has two key functions, one is feature operation which achieves parameters of local operation, and the other is model constraint operation which determines constraint relations between new features and old ones. Function toNSFT() transforms different data structure between variant CAD systems into structure stored by feature dependence graph. Function toLSMC() works like toNSFT()'s inverse process.

\section{B. neutral semantic transition library}

Because of lacking of unified standard in CAD software development, different CAD software have some differentiations from modeling command in high level to data structure in low level, so it makes that mappings between commands in heterogeneous CAD system have various situations.

Many mapping between different systems are realized using a neutral semantic transition library in this paper. Command mapping between different systems have four cases: single operation versus single operation, single operation versus multiple operations, multiple operations versus single operation and $n$ operations versus $m$ operations. Structure of operation mapping is shown in Fig.2.

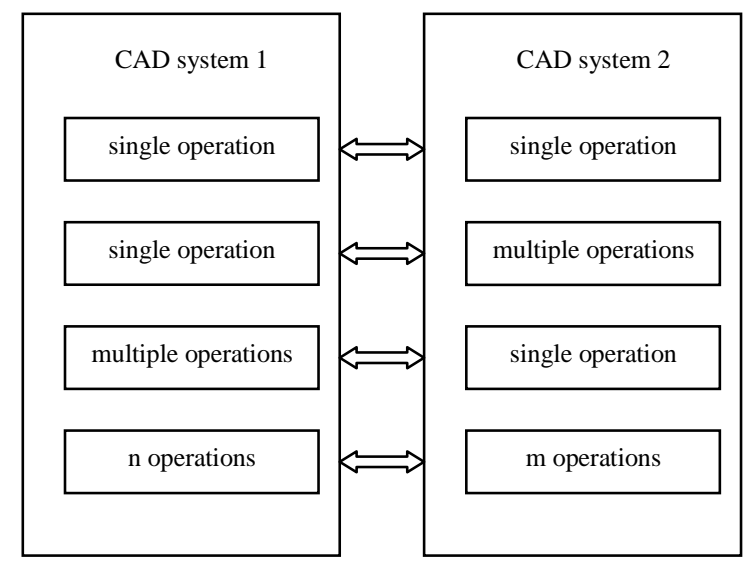

Figure 2. Structure of operation mapping

Neutral semantic transition library should support effectively the four cases. Single operation to single operation is a simple one to one mapping relation, and it is implemented easily in this system. But other three cases are complicate, and there are some difficult in the process of implement. The three kinds of mapping are introduced as follows.

Each NSFT command has a serial number in the neutral semantic transition library, and each different NSFT command has only a command number. Command numberings are corresponding of mapping relations between commands, and have also four cases: single number to single number, single number to a number group, a number goup to multiple numbers and a number group to a number group. Data structure in neutral semantic transition library are shown in Fig.3. 
Single-Single:

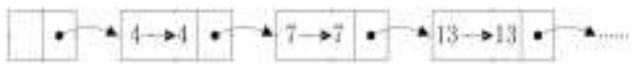

Single-Mul:

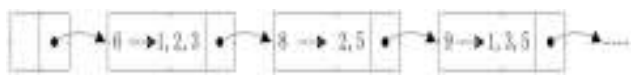

Mul-Head :

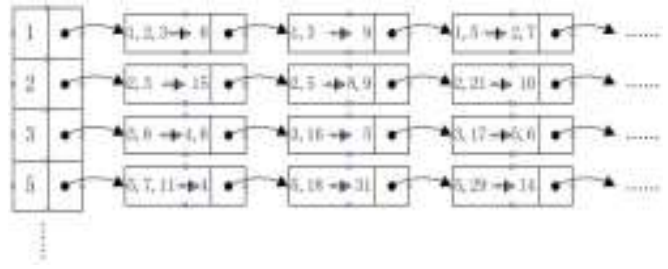

Figure 3. Data structure in neutral semantic transition library

As shown in Fig.3, command numbers stored in linked list are stored according to numbers increase in sequence. Single operation to single operation and single operation to multiple operation are stored in Single-Single linked list and Single-Mul linked list respectively. When searching in Single-Single linked list or Single-Mul linked list, if there is no command to find, then system should search in MulHead linked list. Command numbers of multiple operation to single operation and $n$ operation to $m$ operation are stored in Mul-Head linked list. Each sub-linked list of Mul-Head linked list is stored according to numbers increase in sequence.

After a command number is picked by neutral semantic transition library, it will do three steps:

Step 1: command number picked is stored in mapping node.

Step 2: this stage has five sub-steps:

1. System will search in Single-Single linked list to make sure whether there are command numbers which are consistent with command numbers picked. If yes, then stop searching, and jump to 3 .

2. If no, system will search in Single-Mul linked list. If yes, then stop searching, and jump to 3 .

3. If no, system will search in Mul-Head linked list, and jump to 4 .

4. Waiting for a next NSFT command sent by opposite CAD system, after accepting command numbers, system will search in sub-linked list which has found head numbers. If yes, updating node information, and jump to 4 .

5. If no, current NSFT command is stored in being handling node, then jump to 3 , command number in being handling node is copied to mapping node, and jump 1.

Step 3: command numbers stored in mapping nodes are freed.

Using a multiple operations to single operation as an example is to illustrate this mapping process. Design users who work in HUSTCAID system would stretch a circle to a cylinder, the first step is structure loop operation, after this operation is transformed by neutral semantic feature converter, the operation is sent to UG system site, and the command number 2 is picked by neutral semantic feature converter. Head numbers which consistent with number 2 are searched firstly in Mul-Head linked list, then system wait for a next NSFT command number, namely, stretch operation number 3. After accepting the number 3, system will search in sub-linked list whose head number is 2 . If yes, then updating mapping nodes and system continue picks a next command number. If the command number picked is 7 again, after not finding a number group including number $2,3,7$, then command number 7 will be stored in being handling node, and number group including number 2,3 will be mapped to number 15 , number 15 is transformed to a neutral semantic convert command and is transformed to a local modeling command by neutral semantic feature converter, namely stretching operation.

In a heterogeneous collaborative system, maybe there are many different CAD system, so each system configuration may has different neutral semantic transition library according to differentiation between systems, only in this way, data mapping and transmission between any two different systems will be implemented.

\section{NEUTRAL SEMANTIC FEATURE CONVERSION OF SINGLE PART}

Many basic features are combined by constraint relations in a single part, and UML class chart of a single part is shown in Fig.4.

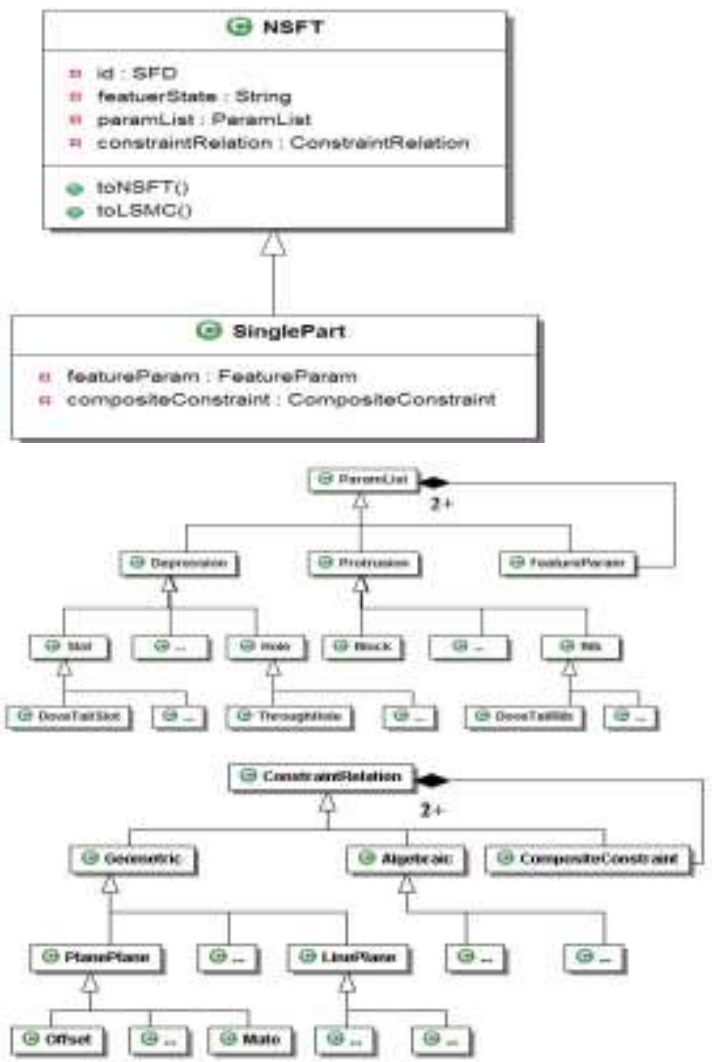

Figure 4. UML class chart of a single part

To a single part, firstly, all parameters of the part are picked by neutral semantic feature converters. Secondly, a 
single part is decomposed into many basic feature, and these basic feature are transformed into neutral semantic feature conversion object in a order. At last, neutral semantic feature conversion objects are listed in a queue in a preferred order, and are sent to other sites in this order. After other sites receive the neutral semantic feature conversion command group, neutral semantic feature conversion object are executed as local modeling command according to basic feature command.

\section{SYSTEM IMPLEMENTATION}

Neuter semantic feature conversion method proposed in this paper has been applied in synchronous collaborative design between HUSTCAID system and UG system. A collaborative modeling interface between HUSTCAID and UG is shown in Fig.5 and Fig.6. Master server site, HUSTCAID site and UG site are configured respectively on three computers in this collaborative system. Client users need only focus on collaborative work on local CAD system, and servers response to clients' requests, so heterogeneous $\mathrm{CAD}$ collaborative design work is implemented in this way.

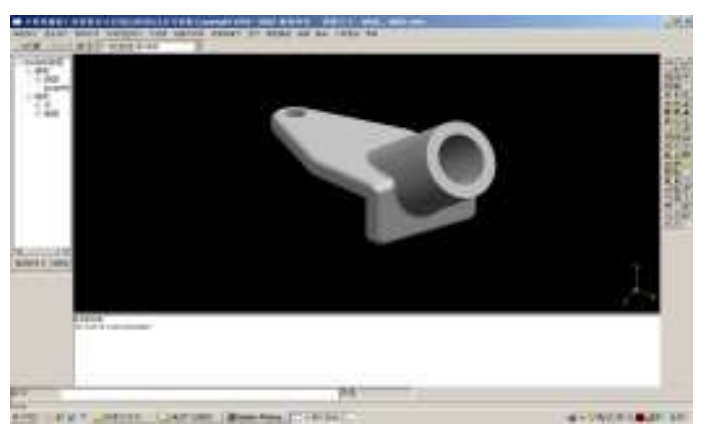

Figure 5. HUST-CAID system interface

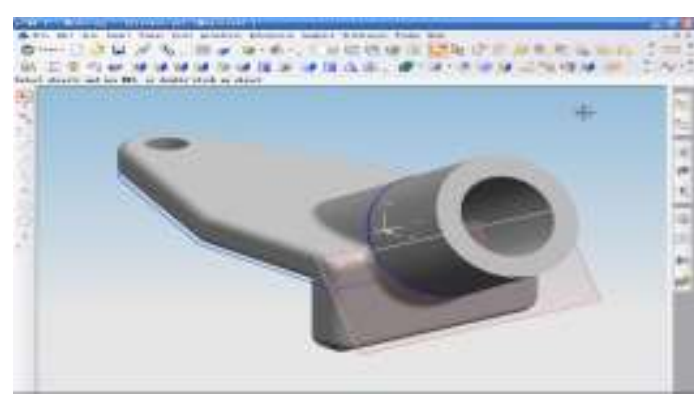

Figure 6. UG system interface

\section{CONCLUSIONS}

Semantic feature modeling technology based on cellular is used to structure CAD models in this paper, this technology overcomes some shortcomings such as semantic description incompletion in the process of modeling, and is applied more suited in the process of synchronous collaborative design. The way that transmit neutral semantic information by features dependence graph can reduce network load and ensures collaborative design work efficiency. System implemented in this paper indicated that synchronous collaborative system of heterogeneous CAD proposed can make users work in a environment of real-time collaborative, and can improve product quality and reduce product design cycle.

\section{ACKNOWLEDGMENT}

This paper is supported by Scientific Research Fund of Heilongjiang Provincial Education Department (NO: 12531571).

\section{REFERENCES}

[1] Henrik Artman, Robert Ramberg, Hillevi Sundholm. A Case Study on Collaborative Design Learning [C].Computer Supported Collaborative Learing,2005 : 603-638.

[2] Xiang Chen, Min Li, Shuming Gao. A Web Services Based Platform for Exchange of Procedural CAD Models[C].The 9th International Conference on CSCW in Design,2005 : 605-610.

[3] Bidarra R, Neels W J, Bronsvoort W F. Boundary Evaluation for a Cellular Model[C]. Computers and Information in Engineering Conference, Chicago, USA, 2003 ; (1) : 255-265.

[4] Bidarra R, Bronsvoort W F. Semantic Feature Modeling[J]. Computer Aided Design, 2000 ; 32(3) : 201-225.

[5] Bronsvoort W F, Bidarra R, Nyirenda P J. Developments in Feature Modeling $[\mathrm{J}]$. Computer-Aided Design and Application, 2006, 3(5): 655-664

[6] Sun Lijuan and Liu Xianguo. Research on Algorithm of Computing Parameter Interval in Geometric Models[J]. Information Technology Journal, 2011, 10(2): 402-408.

[7] Den Berg E V, Bronsvoort W F. Validity Maintenance for Freeform Feature Modeling $[\mathrm{J}]$. Journal of Computing and Information Science in Engineering, 2010, 10(1): 1-14.

[8] Liu Xianguo, Sun Lijuan, Wang Qihua and et al. Research on Family of Object Model in Semantic Feature Modeling[J] Computer Science, 2011, 38(3): 286-289.

[9] Nick, J., Tuecke, S.: Sport and Economic Regeneration in Cities. Urban Studies, pp. 985--999 (2005)

[10] Foster, I., Kesselman, C.: Old Locals and New Spaces. Leisure Studies, vol.24, pp.415--434 (2005)

[11] Lin, X.: Research on the Construction and Post-Games Utilization of Modern Olympic Venues. Journal of Beijing Sport University, vol.28, pp.1441--1444 (2005)

[12] Luo, P.: Research of the Management Profit of Large Sports Entertainment Yards in China. Journal of Xian University of Physical Education, vol.24, pp.17--20 (2007)

[13] Steinke, T.: Olympic Games Host City Marketing: An Exploration of Expectations and Outcomes. Sport Marketing Quarterly, Vol. 12, pp.37--47 (2003) 